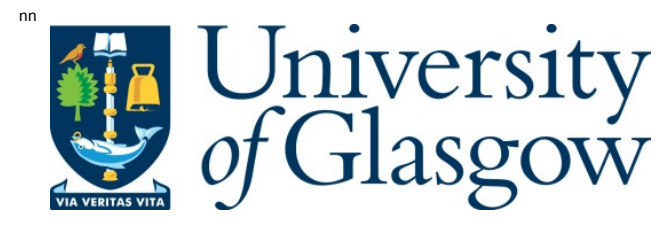

M orris, B. J ., and Pratt, J. A . (2014) Novel treatment strategies for schizophrenia from improved understanding of genetic risk. Clinical Genetics, 86 (5). pp. 401-411.

Copyright @ 2014 J ohn Wiley \& Sons, Ltd.

A copy can be downloaded for personal non-commercial research or study, without prior permission or charge

Content must not be changed in any way or reproduced in any format or medium without the formal permission of the copyright holder(s)

http://eprints.gla.ac.uk/101899/

Deposited on: 02 February 2015

Enlighten - Research publications by members of the U niversity of Glasgow http://eprints.gla.ac.uk 


\title{
Novel treatment strategies for schizophrenia from improved understanding of genetic risk
}

Brian J.Morris $^{1,2}$ and Judith A.Pratt ${ }^{1,3}$.

1. Psychiatric Research Institute of Neuroscience in Glasgow (PsyRING), University of Glasgow, Glasgow, G12 8QQ, UK; 2. Institute of Neuroscience and Psychology, School of Medical, Veterinary and Life Sciences, University of Glasgow, Glasgow, G12 8QQ, UK; 3. Strathclyde Institute of Pharmacy and Biomedical Sciences, University of Strathclyde, Glasgow, G4 ONR, UK

Author for correspondence:

B.J.Morris,

PsyRING,

West Medical Building,

University of Glasgow,

Glasgow, G12 8QQ, UK

email: Brian.Morris@glasgow.ac.uk

phone: 44-141-330-5361

fax: 44-141-330-5659

\section{Conflict of interest}

The authors have declared that there are no conflicts of interest.

\begin{abstract}
Recent years have seen significant advances in our understanding of the genetic basis of schizophrenia. In particular, genome-wide approaches have suggested the involvement of many common genetic variants of small effect, together with a few rare variants exerting relatively large effects. While unequivocal identification of the relevant genes has, for the most part, remained elusive, the genes revealed as potential candidates can in many cases be clustered into functionally-related groups which are potentially open to therapeutic intervention. In this review, we summarise this information, focussing on the accumulating evidence that genetic dysfunction at glutamatergic synapses and post-synaptic signalling complexes contributes to the aetiology of the disease. In particular, there is converging support for involvement of post-synaptic JNK pathways in disease aetiology. An expansion of our neurobiological knowledge of the basis of schizophrenia is urgently needed, yet some promising novel pharmacological targets can already be discerned.
\end{abstract}




\section{Introduction}

Schizophrenia imposes an enormously heavy burden on sufferers, carers, and society. The disease has the doubly adverse characteristics of being both common and serious. Lifetime risk of developing the disease is around $0.8 \%$, and from the time of first appearance of the symptoms (typically in late adolescence or early adulthood) the majority of patients will be unable to make a full recovery, with many committing suicide (1).

Patients can suffer from a range of symptoms. These are typically classified as positive (in the sense of being additional to normal experience) such as delusions and hallucinations (most commonly auditory, but also including other sensory modalities); negative (in the sense of being missing from normal experience) such as lack of motivation, self-neglect, and social withdrawal; and cognitive, including impaired ability to sustain attention, deficits in working memory, and loss of cognitive flexibility. The range of symptoms experienced can be quite variable.

Environmental factors are known to influence the risk of suffering from schizophrenia. Known risk factors are clustered around antenatal and perinatal periods, and include antenatal stress, malnutrition or viral infection, and perinatal hypoxia. These environmental risk factors interact with a substantial level of inherited genetic risk (that raises prevalence to around $50 \%$ in monozygotic twins). The mechanistic basis for these gene - environment interactions is not understood, but may become clearer once the genetic influences on the disease are better-characterised.

Current drug treatments rely on antagonism of the D2 dopamine receptor, generally incorporating a spectrum of actions at other G-protein coupled receptors (GPCRs) that modify the drug's efficacy and side-effect profile. Inhibition of D2 dopamine receptors produces a gradual reduction in the severity of positive symptoms in the majority of patients, without much improvement in negative or cognitive symptoms. The reduction in positive symptoms is immensely valuable, yet the lack of efficacy against other symptom domains, the significant proportion of patients who are treatment-resistant, and the widespread and unpleasant side-effect profile of the drugs, makes the discovery of improved therapeutic strategies of paramount importance. Rational drug development programmes based on a coherent understanding of genetic risk mechanisms offer the possibility of treating the neurobiological dysfunction that causes the disease, rather than alleviation of symptoms. As will be discussed further below, a large number of genes are believed to contribute to genetic risk of schizophrenia, and it will be crucial to identify the optimum targets for pharmacological intervention. This will need to consider both the extent to which modulating the target can restore function of the biochemical network to normal, and also the target's pharmacological tractability. Naturally there are different opinions as to which proteins (or RNAs) represent viable targets for drug development; however, there are some widely-held principles that are supported by general experience (S1, S2) (see list in supplementary information for S- citations). Thus GPCRs, ion channels, membrane transporters and cytoplasmic enzymes are the most common drug targets at the moment, with nuclear receptors, proteases and protein kinases also successfully exploited for therapeutic purposes. We emphasise these classes of protein in subsequent sections.

\section{Genetics of schizophrenia}

Psychiatric genetics researchers have migrated over a period of time from cytogenetics, linkage and candidate gene association studies, to genome-wide association studies (GWAS) with ever-increasing 
sample sizes, and genome-wide studies of copy number variation (CNV), through to whole-exome and whole-genome sequencing. Genetic overlap with other psychiatric diseases, including autism spectrum disorders and intellectual disability, has been a notable finding. In relation to schizophrenia, cytogenetic studies have most notably identified a translocation disrupting the DISC1 gene as a high-penetrance risk factor for psychiatric illness (2). Linkage studies have reliably detected chromosomal loci linked to increased risk of the disease, and candidate gene studies have proposed quite a large number of individual genes as contributing to elevated disease risk. In many of these cases, the reported associations have not stood the test of replication in independent sample cohorts, although in some instances (mentioned below where appropriate), data from GWAS or CNV studies have supported the original findings. Importantly, CNV and GWAS have drawn attention to a number of new candidate genes. A few robust associations have been discovered, despite the fact that significance thresholds are generally demanding, to avoid impossibly high type I (false positive) error rates. It should be remembered that less attention is generally paid to the corresponding potential for type II (false negative) errors (S3), so the lack of a GWAS hit should in no way diminish credence in the importance of a gene, if supported by strong evidence from other approaches. Deep sequencing studies of complete exomes (the entire mRNA sequence) from patients is now revealing further sequence variations/mutations that, while rare, shed light on the neurobiological dysfunction that underlies the common disease.

Current knowledge about the genetic basis of schizophrenia has been the subject of a number of excellent recent reviews $(3)(S 4, S 5)$. Hence, rather than covering the same ground, the current review aims to highlight specifically where advances in genetic understanding are suggesting novel approaches to drug design and development. We focus on the genes and gene families where the genetic support is relatively strong, and where the possibilities for pharmacological intervention are relatively good. Hence a number of genes where evidence could be seen as really convincing may be beyond the scope of the review if there is no obvious route for drug development at present. For example, the MHC region of chromosome 6p21-22.1 has been robustly associated with schizophrenia in GWAS, but is not obviously amenable to drug development according to current understanding, and therefore is not discussed here. In addition, by following a holistic approach, we bring forward emerging and converging neurobiological themes in the hope that they may illuminate the darker areas of disease aetiology.

As a brief summary of the present situation, it would be fair to say that while the general features of the genetic landscape of schizophrenia are now known, it has proved difficult to resolve much specific detail $(\mathrm{S} 4, \mathrm{~S} 5)$. Current understanding suggests that a large number of common gene variants, generally having a relatively small effect on disease risk individually, combine with a smaller number of rare variants, which individually have a much larger influence. In general, CNVs tend to increase disease risk to a much greater extent than individual SNPs (4-6)(S6). In addition, the effects of these inherited genetic risk factors are likely to be further augmented by a small number of de novo mutations unique to the affected individual. Hence even where susceptibility to schizophrenia seems to be strongly inherited within a family pedigree, the actual genetic inheritance may be quite complex. It follows that, in order to gain an overview of how genetic influences lead to the development of the disease, and hence how novel therapeutic approaches might be designed, it is essential to consider a potentially large number of genes, most likely with groups of them clustered in a fairly intimate functional relationship, interacting with environmental and neurodevelopment factors. This is clearly no simple task, and while some patterns are emerging in terms of important functional gene/protein clusters, the ways in which sequence variations in specific genes interact to cause the disease remain to be clarified. Equally, despite the intuitive expectation that symptomatic variation might reflect the polygenic nature of the disease, there is little information at 
present on the relevance of individual genetic variants to specific symptom domains. It might be thought that, due to their relatively large effects, the genes within CNVs might be particularly informative for understanding disease aetiology. However, in nearly every case, the CNV affects a number of genes. Hence the substantial increase in risk of schizophrenia due to a particular CNV cannot be assigned unequivocally to a specific gene. Table 1 summarises the extent to which genes within the CNVs fit with our growing understanding of the patterns of neurobiological dysfunction in schizophrenia.

\section{Glimpses of patterns}

It can be argued that some definite features of the neurobiology of schizophrenia can at last be discerned through the swirling mists of phenotypic and genetic heterogeneity. In general, the more reproducible findings from candidate gene studies have supported pharmacological and biochemical evidence that glutamate synapses might be particularly affected in schizophrenia $(7,8)$. GWAS, CNV and, very recently, exome sequencing studies have further strengthened this idea $(4,9-12)$. The relevant genes and supporting data are summarised in Figure 1, and detailed below. A picture of compromised post-synaptic signalling processes is also emerging, where the risk genes encode clusters of functionally-related scaffolding proteins and kinase cascades that integrate and transduce synaptic events $(4,10,11,13)$. Prominent among these are proteins involved in the c-Jun $\mathrm{N}$-terminal kinase (JNK) signalling pathway - a multi-level kinase cascade involved in both physiological and pathological plasticity in neurones (14). Of course this may link extremely well with the evidence for impaired glutamate synaptic function. These results are summarised in Figure 2 and explained in more detail below, under consideration of functional classes of potential therapeutic target. It must be emphasised that the evidence is seldom unequivocal. A number of different hypotheses have been proposed to explain the heterogeneous genetic evidence as it emerges. However, compromised function of glutamate synapses and downstream signalling pathways is arguably by far the strongest unifying (or at least partially unifying) hypothesis. In the following sections we have attempted to sift through the abundant harvest of genetic information, to separate those grains of knowledge with the potential to form the seeds for future drug development. We describe how genetic evidence has brought us to this position, and indicate the novel therapeutic avenues that are now attractive for exploration.

\section{Ligand-gated ion channel receptors}

A number of studies have investigated possible association of NMDA receptor (NMDA-R) subunit genes SNPs with schizophrenia (e.g. S7,S8) However, where an effect has been detected, these findings have not always proved replicable. Overall there may be at best a hint that NMDA-R subunit gene sequence variations contribute to disease risk. In recent genome/exome-wide studies, patient samples were enriched for common GRIN2A variants (12) and rare nonsynonymous de novo GRIN2A mutations (4), while a missense GRIN2B variant has been detected in a patient (S9). Some cytogenetic evidence suggests that disruption of the GluK4 (GRIK4) kainate receptor subunit gene may be associated with schizophrenia, and some but not all case-control association studies support this (15). SNPs in the GRID1 gene, encoding the delta 1 ionotropic glutamate receptor subunit, were found to be associated with schizophrenia in two studies (S10,S11), and the gene is within the 10q23 duplication region that shows high penetrance for schizophrenia (Table 1). However, the function of delta ionotropic glutamate receptors is not clearly understood, so the therapeutic relevance of these findings is not clear. The scaffolding protein PSD-95 is highly abundant in the post-synaptic density and plays a key role in glutamatergic signalling, but there is no strong evidence implicating the corresponding gene in schizophrenia. However, there is evidence in the case of the DLG genes, which encode closely related proteins. For example, DLG1 encodes a PSD-95related protein involved in various scaffolding functions including AMPA and kainate receptor trafficking. 
Rare DLG1 mutations have been found in patients (11) along with microdeletions that include the DLG1 $(16,17)$ (Table 1$)$ or DLG2 $(18)$ gene. This supports the overall concept of glutamate synapse/signalling dysfunction in schizophrenia, and drugs targeted at facilitating NMDA-R function have so far proved mildly promising (S12). With the knowledge that different types of NMDA-R (for example GRIN2Acontaining vs GRIN2B-containing) can have markedly different functions, there are as yet unexplored therapeutic possibilities in this area.

Similarly, early association studies focussed on genes encoding subunits of the $G A B A_{A}$ receptor did not produce conclusive findings. However, the presence of the GABRD gene (encoding the delta subunit) in the 1 p36.33 duplication, and a cluster of genes encoding the alpha 5 , beta 3 and gamma 3 subunits within the 15q11-q13 duplication, both associated with schizophrenia (Table 1) is noteworthy. GABA $A_{A}$ receptors containing alpha 5 subunits and delta subunits have been specifically linked to the regulation of $\gamma$ oscillatory EEG activity (S13), which is perturbed in schizophrenia (19). The potential of $\mathrm{GABA}_{A}$ receptor modulating drugs for treating schizophrenia might therefore be worth some further attention, although this particular route is quite well-travelled (20).

The overall strength of evidence is quite compelling for the $\alpha 7$ subunit of the nicotinic acetylcholine receptor. There is a highly significant association of deletions at the $15 q 13.3$ locus with schizophrenia (21). The CHRNA7 gene (encoding subunits found in the $\alpha 7$ subtype of nicotinic receptor) is one of 12 genes within the deletion (Table 1), and is probably the strongest candidate for elevating disease risk. Other nicotinic receptor subunit genes (CHRNA3, CHRNA5) may also be implicated, from the most recent GWAS data (12). Some involvement of nicotinic receptors with schizophrenia is suggested by the fact that the majority of patients are smokers, possibly as a form of self-medication. Stimulation of nicotinic $\alpha 7$ receptors acts to enhance glutamate release in areas such as the cerebral cortex, hippocampus and thalamus (Fig 1), and reduced levels of nicotinic $\alpha 7$ receptors have been noted in post-mortem studies of thalamic tissue from patients (S14). Clinical trials report that nicotinic $\alpha 7$ receptor agonists show possible beneficial effects on negative and cognitive symptoms (22).

\section{Voltage-gated ion channels}

$\mathrm{Ca}^{2+}$ channels are clearly important for many aspects of neuronal excitability and communication. $\mathrm{N}$-type $\mathrm{Ca}^{2+}$ channels are predominantly presynaptic (Figure 1), although not only on glutamatergic terminals, while T-type and L-type channels are widespread with a mainly post-synaptic location, and are not predicted to affect any specific neurotransmitter system preferentially. The CACNA1C gene encodes the pore-forming $\alpha 1$ subunit which, along with accessory $\alpha 2, \delta-1$ and ${ }^{2}$ subunits, produces L-type (Cav1.2) voltage-dependent $\mathrm{Ca}^{2+}$ channels. Sequence variations in the CACNA1C gene, most prominently linked to Timothy Syndrome, have been robustly associated with bipolar disorder, and now seem to be reproducibly associated with schizophrenia as well $(12,23,24)(S 16, S 16)$. Sequence variations in the gene encoding the channel's ${ }^{2}$ subunit - CACNB2 - may also show significant genome-wide association with schizophrenia $(12,25)(S 17)$. Rare mutations in voltage-gated calcium ion channel genes, including CACNA1C, were also found in another recent exome sequencing study (11). Similar evidence is now being reported for other closely-related genes. De novo mutations have been detected in the CACNA1I, encoding the alpha subunit of the T-type voltage gated calcium channel (S18), a gene which has also been identified in the most recent GWAS (12), and deletions close to the CACNA1B gene, encoding the $\alpha 1$ subunit of $\mathrm{N}$-type (Cav2.2) $\mathrm{Ca}^{2+}$ channels, have been detected at higher frequency in patients compared to controls (9). The genetic evidence for voltage-dependent $\mathrm{Ca}^{2+}$ channel impairment is therefore reasonably convincing at the moment. However, our neurobiological understanding of the nature of this impairment in schizophrenia, and how it might be ameliorated, has not kept pace with the genetic advances. 
Nevertheless, small molecules targeting voltage-dependent $\mathrm{Ca}^{2+}$ channels are under development for epilepsy and pain, in addition to cardiovascular applications, so there is definite potential here.

\section{G-protein-coupled receptors (GPCRs)}

GPCRs are of course attractive as novel targets for drug development. Candidate gene studies focussed on the mGlu3 (GRM3) metabotropic glutamate receptor have produced conflicting results, and no clear picture has emerged, although an association signal was recently detected by GWAS (12). Clinical trials of a mGlu2/3 agonist looked promising initially (26) but are still not conclusive in the light of follow-on trials (27). In the case of the mGlu5 (GRM5) metabotropic glutamate receptor, a rare mutation has been noted in familial schizophrenia (28).

Rare microduplications involving the VIP/PACAP VPAC2 receptor (VIPR2) have been detected at significantly higher frequency in patients compared to control subjects $(29,30)(\mathrm{S} 19)$, but see also(31). The VPAC2 receptor can couple to various signalling processes, most commonly via adenyl cyclase and phospholipase C (S20). There is no obvious reason why increased gene dosage at this receptor should increase risk of schizophrenia, but better understanding of the neurobiology of VPAC2 and its relationship to psychiatric disease might well lead to novel therapeutic approaches. We note also that the type I $\mathrm{CRH} / \mathrm{CRF}$ receptor gene (CRHR1) is located at the site of the rare 17q21.31 microduplication detected in some patients with schizophrenia (5). Interneurones expressing both VIP and CRF (S21) may play an important role in the regulation of prefrontal cortex pyramidal neurones, so a pharmacological strategy based on these neuromodulatory GPCRs deserves consideration.

\section{Type I transmembrane proteins}

The least ambiguous genetic evidence for a role in schizophrenia concerns the neurexin 1 (NRXN1) gene. Deletions encompassing exons of the NRXN1 gene are found at higher frequencies in patient samples than in control groups $(9,29)$, and there is the possibility that rare mutations could also play a role as well as functional deletions (32)(S9). It is interesting that a possible signal for a trans-synaptic binding partner of neurexin 1 - neuroligin 4 (encoded by the NLGN4 gene) - has been detected in GWAS $(12,25)$ (Figure 2). Neuroligin-neurexin binding spans the synaptic gap (Figure 1), contributing to synapse development and maintenance (S22). A loss of this interaction can be rationalised in terms of theories of synaptic dysfunction in schizophrenia. Although maybe not the simplest drug target, there is great diversity/specificity in the neurexin-neuroligin binding interaction (S23) that may be open to exploitation pharmacologically.

The low-density lipoprotein (LDL) receptor gene family includes the low-density lipoprotein receptorrelated proteins 1 and $1 \mathrm{~B}$ ( $L R P 1$ and $\angle R P 1 B$ ) genes. They encode closely-related type I transmembrane glycoproteins, best-known for a role in cholesterol trafficking and ApoE binding. However, they also interact with NMDA-Rs via the post-synaptic density (S24), and act to suppress JNK activity (S25). One of the earliest exome sequencing studies in schizophrenia identified a de novo mutation in LRP1 in a proband (33). The LRP1 locus has also been detected in recent GWAS (12). It is interesting that nonsynonymous sequence variations in LRP1B were detected in samples from patients but not control subjects in another exome sequencing study (34), and also in a family with a high incidence of schizophrenia (28). These intriguing results need neurobiological support to underpin a potential role in schizophrenia aetiology, but they may in the future provide a foundation for novel therapeutic approaches. 
ERBB4 is a receptor tyrosine kinase belonging to the epidermal growth receptor family. Both ERBB4 and its ligand - neuregulin, have been the focus of considerable attention in candidate gene association studies (S26). In addition, the Neuregulin 3 gene (NRG3) is within the 10q23 duplication region associated with schizophrenia (5). In GWAS, ERBB4 SNPs generally show some degree of association without achieving genome-wide levels of significance $(25,35)$, yet rare deletions encompassing the ERBB4 gene have been detected in patient samples $(18,21)$. Small molecule inhibitors such as gefitinib are active on ERBB4 without being selective, yet show the potential for modulating ERBB4 signalling in schizophrenia.

MERTK - a type I transmembrane tyrosine kinase which has recently been implicated in synapse elimination (S27), can activate a variety of signalling pathways, including Rac, ERK and JNK (S28). Duplications including MERTK that cosegregate with schizophrenia have recently been identified at a schizophrenia-linked locus (36)(S29). As with ERBB4, small molecule inhibitors are being developed (S30), so again there is potential in this route if the connection between MERTK and schizophrenia was consolidated.

\section{Transporters}

The SLC1A1 gene encodes a glutamate transporter (EAAC1 / EAAT3) responsible for the uptake of synaptic glutamate into neurones (Fig 1). A deletion including exons of the SLC1A1 gene was enriched in the patient group of a large case-control sample (37), cosegregated with schizophrenia in a Palauan family(38), and has been found in another patient with schizophrenia. Similarly, a rare deletion of 4 genes including another glutamate transporter gene, SLC1A3, was detected in a patient cohort $(18,36)$. This might be tentative evidence that a developmental deficit in glutamate uptake may predispose towards schizophrenia.

\section{Phosphodiesterases}

PDE4B is a phosphodiesterase involved in the termination of CAMP signalling. PDE4B is an interacting partner of DISC1, and a chromosomal translocation disrupting PDE4B has been noted in subjects with schizophrenia (39). While there is a general issue of nausea with PDE4 inhibitors, isoform-selective agents may have considerable promise.

\section{Rho GTPases}

Small GTPases of the Rho family play an important role in the CNS in the regulation of neuronal morphology and axon growth. Prominent members of the family such as RhoA, RhoB, Rac 1-3 and cdc42 are all highly expressed in the brain and are stimulated by synaptic activity $(40,41)$. Their activity is tightly regulated by GTPase activating proteins (GAPs), guanine nucleotide exchange factors (GEFs) and guanine dissociation inhibitors (GDIs).

The GTPases themselves have not shown up prominently in any genetic studies, with the possible exception that the there is a tentative GWAS signal relating to the control of expression of the CDC42 gene (S31) (Figure 2). However, there is a variety of suggestive evidence relating to Rho GTPase regulatory proteins. Potentially pathogenic sequence variations in the p164-RhoGEF (ARHGEF17) and TIAM2 genes were detected in samples from patients but not control subjects in an exome sequencing study (34). TIAM2 is interesting, as SNPs in this gene were also detected in a GWAS (S32). The TIAM2 protein is is a Rac GEF, is part of the DISC1 interactome (42) and also binds NRXN1 (S33) - one of the genes mostunequivocally associated with schizophrenia, as noted above (Fig 1). TIAM2 is also interesting in that it has a remarkable forebrain-specific pattern of expression (S34). Evidence suggests that TIAM2 functions 
mainly as an activator of Rac signalling. Furthermore, the CYFIP1 gene - one of few genes located within the 15q11.2 deletion region (Table 1) - encodes cytoplasmic FMR1 interacting protein 1/Rac1-associated protein 1, which interacts with Rac1 as part of a complex regulating synaptic morphology and axon growth (S35). An intronic SNP in the CYFIP1 gene also shows association with schizophrenia (43). Thus there is a cluster of genetic evidence suggesting possible dysfunction in Rac signalling. While it probably also has post-synaptic actions, Rac1 has a prominent role in axon growth and presynaptic vesicle release $(\mathrm{S} 36, \mathrm{~S} 37, \mathrm{S38})$, and hence we show it presynaptically in Figure 1.

Three further GEFs also deserve mention. A significant association signal and possible rare mutations in patients have also been detected for the KALRN gene (S39) - encoding kalirin - a Rho/Rac1 GEF that is located at synapses and interacts with DLG1 and DISC1, while a de novo mutation in DOCK1 (DOCK180 - a Rho family GEF) was detected in a patient with schizophrenia (44). DOCK180 is involved in axon growth and synapse stabilisation, and interacts with adaptor/scaffold proteins such as NCK1 and CRKL to transduce signalling information (S40) (Figure 2). Equally, the gene encoding a GEF for a related GTPase (Rap2) - PDZ-GEF1 (RAPGEF2) is close to microdeletions found in patients (9) and is within a duplication segregating with disease in an Afrikaner family (45). Of course these very rare genetic events do not in themselves demonstrate a causal role in schizophrenia aetiology. However they are consistent with a picture of GTPase signalling impairment in the disease.

Indeed, there is also noteworthy evidence implicating Rho GAPs in genetic risk for schizophrenia. The ARHGAP11B gene lies within the 15q13.3 deletion locus (29), but unfortunately the function of this gene is poorly characterised. Microduplications (3p25) in the region of the SRGAP3 (ARHGAP14) gene, which encodes another Rac1 modulator, have been reported to cosegregate with schizophrenia and related disorders (S41). Duplications in this region were also detected in patient samples in a genome-wide study (46). Apart from its action as a Rac suppressor, SRGAP3 is a direct TNIK interactor (see next section), and hence potentially linked to the DISC1 interactome (42). In addition, suggestive evidence involving the closely related ARHGAP4 gene, encoding SRGAP4, was obtained in a recent GWAS (47). The ARHGAP1 (cdc42GAP) locus has also been detected in recent GWAS (12)(S42). The gene encoding Brain-Specific Rho GTPase-activating protein RICS/GRIT/GC-GAP (ARHGAP32), a Rho GAP (S43), was found to be associated with schizophrenia in a candidate gene study (48). RICS/GRIT binds to CRKL (a scaffold protein whose gene lies within the 22q11 deletion region; Figure 2, and see below), the adaptor protein NCK1, as well as CDC42, and is activated by NMDA-R stimulation (S44). The Ras GAP SYNGAP1 may also be important. Enrichment of synaptic function genes (including SYNGAP1), was found in another recent exome sequencing study searching for rare mutations (11) and a de novo mutation in SYNGAP1 was detected in a patient with schizophrenia (44).

Rho signalling in general is pharmacologically tractable, due to the diversity of regulatory molecules, their relative functional specificity, and the possibilities for either reducing or increasing pathway activity with small molecule inhibitors by targetting either GEFs or GAPs. On the other hand, the plethora of interactions and feedback loops can complicate the delivery of a sustained therapeutic effect. However, small molecule inhibitors of Rho GTPase signalling are currently being developed (S45,S46), and if the exact details of the dysfunction in these pathways in schizophrenia could be determined, the therapeutic potential might be considerable.

\section{Kinases and phosphatases}


Rac1 can activate a number of downstream signalling processes, but prominent among them are the Rac/Cdc42/p21-activated kinases (PAKs). Three PAK genes are implicated in genetic susceptibility to schizophrenia: microdeletions at a locus including PAK2 have been observed at increased frequency in patient cohorts $(16,17,29)$, rare PAK7 duplications have also been detected (S47)(46), and common variants at the PAK6 locus have been detected in recent GWAS(12). This supports the concept of a dysfunctional Kalirin/SRGAP3/Tiam2 - Rac -PAK2/PAK6/PAK7 pathway, although whether the end result is too little or too much activity is not clear. While not extensively investigated, there is some evidence of a presynaptic role for PAK2 and PAK7 (also known as PAK5) (S48,S49,S50). Inhibitors of PAKs have recently been reported to ameliorate neurobiological deficits in mice with compromised Disc1 function(49), so there is support for the idea that this could be a useful way forward.

A rare duplication encompassing the $P R K C D$ gene, encoding the delta isoform of protein kinase $C$, and a rare non-synonymous sequence variant in the $P R K C B$ gene, encoding the beta isoform of protein kinase $C$, have been detected in patient samples $(18,44)$. An significant association close to the Nu type Protein Kinase C (PRKD3) gene has also been detected (25), so there is a little evidence of PKC isoform involvement in the disease, that at present is far from being conclusive.

TNIK (TRAF2 and NCK-interacting kinase) is a MAP kinase kinase kinase kinase (MAP4K) originally linked to JNK signalling (S51) and to Rap2 effects (S52) via a NCK1 interaction (Figure 2) but also known to interact with DISC1 (42). TNIK plays a role in glutamatergic synapse regulation and cognitive processes (50-52). A GWAS highlighted TNIK SNPs as potentially associated with metabolic hypofrontality in patients with schizophrenia (53) (S53), and there has been some signal at the TNIK locus in other GWAS studies (35). Interestingly, the NCK1 locus has also been detected in recent GWAS(12). Thus the biochemical, neurobiological and genetic data together are sufficient to make TNIK a potentially interesting target.

Thousand and one kinase type 2 (TAOK2, MAP3K17) is now of substantial interest with respect to novel approaches to treating schizophrenia. Strong evidence now exists that microduplications at $16 \mathrm{p} 11.2$ substantially increase risk of schizophrenia $(O R \sim 8)(54,55) .16 p 11.2$ duplications are also implicated in childhood onset schizophrenia (56). Of the roughly 30 genes within the duplicated region, the prime candidate is TAOK2, since a common variant located within this gene is associated with schizophrenia in GWAS $(O R \sim 1.1)(12,57)$. TAOK2 is a MAP kinase kinase kinase (MAP3K) mediating activation of p38 and JNK, best-characterised for its role in immune responses. While p38 signalling is not compelling implicated in schizophrenia, JNK signalling is starting to look very interesting (Figure 2). It may be relevant that a rare de novo deletion including the closely-related TAOK3 gene has also recently been implicated in schizophrenia (S54).

Another MAP3K, TGF-beta activated kinase 1 (TAK1 / MAP3K7) is similar to TAOK2 in that it is involved in the activation of $\mathrm{p} 38$, JNK and also NFkB signalling in many cell types. While there is no genetic evidence at present implicating the TAK1 gene in schizophrenia risk, TAK1 interacts directly with TAOK2 (S55) and TNIK (S56). In addition, the DUSP14 gene, which encodes a dual-specificity phosphatase (MKP6) that dephosphorylates TAK1 is located within the 17q12 deletion/duplication region associated with schizophrenia (S57) (Table 1).

STK11, also known as LKB1, is a kinase that is deficient in Peutz-Jeghers syndrome. A rare duplication in the gene has been found in patients (58). There is also some evidence from another small study for CNV association with schizophrenia at this locus (S58). AMP-activated protein kinase (AMPK), which is a major 
substrate of LKB1, monitors cellular energy status, and is best-known as a regulator of insulin sensitivity. AMPK is composed of a catalytic alpha subunit, and non-catalytic beta and gamma subunits. PRKAB2 (1q21) encodes the beta2 regulatory subunit, and a deletion at this locus has been noted in patients (Table 1) $(29,59)$. Similarly, a rare PRKAG2 (gamma2 subunit) deletion has been identified in a patient cohort (18), so together this could be viewed as accumulating evidence for dysfunctional LKB1-AMPK signalling in some patients. In fact, AMPK can be activated by both STK11 and TAK1 (S59), and so represents a possible point of convergence for these two pathways (Figure 2).

A de novo mutation in another MAP3K mediator of p38 and JNK signalling - ASK1(MAP3K5) - was recently reported in a patient with schizophrenia (44). This is of interest as a missense mutation in the PPEF2 gene (encoding the protein phosphatase with EF-hand calcium binding domain type 2) was recently identified in a schizophrenia pedigree (28). PPEF2 reduces JNK signalling by suppressing ASK1 activation (S60). Hence the evidence from these mutations is consistent with the importance of upstream JNK pathway components for schizophrenia.

A further kinase involved in JNK signalling - vaccinia-related kinase 2 (VRK2) - is also implicated in schizophrenia from GWAS data (OR 1.1) $(12,60,61)$, and is known to interact with TAK1 and the MAP2K Map kinase kinase 7 (MKK7) $(\mathrm{S61}, \mathrm{S62}$ ), one of two MAP2Ks that control JNK activation, derived from the $M A P 2 K 7$ gene. In fact we recently showed that common variants in the MAP2K7 gene were associated with schizophrenia in two separate cohorts, with one of the largest effect sizes known for a common variant $(O R \sim 1.9)(62)$. There is some supportive evidence from cytogenetic and CNV studies, in that an unconfirmed report suggested that chromosomal rearrangement in this region may represent a high penetrance site for schizophrenia (S63), two studies found nearby markers to show association or linkage

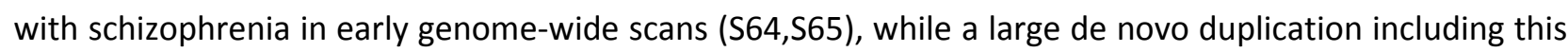
gene was found in a small patient cohort (63). The function of MKK7 in neurones is not particularly well understood, but it contributes to post-synaptic signalling processes and also to the regulation of axonal growth $(14,64)$. A duplication of the MAP2K4 gene, encoding the other JNK-activating MAP2K (MKK4), has been noted in an extended schizophrenia pedigree (S66).

Thus there are signs of disease-relevant genetic variability at multiple points upstream in the JNK signalling pathway. Targetting the JNK pathway in schizophrenia might initially seem risky, due to its contribution to many physiological and pathological processes in many different tissues, and hence the danger of serious side effects. However, in contrast to other MAP kinases, expression of JNKs in the brain shows some degree of preferential localisation to cortical, hippocampal and thalamic areas (65)(S67).

Furthermore, there is a great deal of diversity, particularly at the level of MAP3Ks, MAP4Ks and phosphatases, and the prospects for an adequate level of selectivity seem good if the neurobiology of pathway dysfunction can be understood sufficiently well. The multiple interactions and feedback loops characteristic of MAP kinase signalling pathways (66)(S68) mean that this will be particularly important for successful therapeutic exploitation. Despite the reduced scope for functional selectivity at the level of JNKs, drugs targeting JNKs are already in development for other therapeutic areas (S69).

\section{Functional implications}

It will be apparent from the preceding sections that both RAC1 and TAK1 interact directly with the protein products of many schizophrenia risk genes, while not themselves being genetically implicated in schizophrenia (Figure 3). Their position as hubs within a potentially dysfunctional network may make them especially attractive as target points to restore normal function. Small molecule inhibitors of GEF- 
Rho GTPase interactions are being developed, so pharmacological manipulation of Rac GTPases is not an insurmountable problem. Similarly, selective inhibitors of TAK1 exist (S70), although it is not clear of course whether a potential drug for schizophrenia should reduce or increase activity. Thus these targets are tractable, should further work establish their validity as a way of restoring functional deficits.

\section{Translational implications}

Modelling the aetiology of a polygenic disease in rodents, in order to understand disease neurobiology better, and explore novel therapeutic ideas, is clearly going to be a challenge. At first sight, reproducing one of the high penetrance CNVs in mice by genetic manipulation of the syntenic region would seem like a promising strategy to reproduce aspects of schizophrenia. However, penetrance is incomplete for all CNVs (5)(S71), suggesting that even at a simplistic level, not all GM mice would be expected to show a relevant phenotype. There is also the specific issue that these CNVs are typically linked to intellectual disability as well as schizophrenia. Since the symptom domains of schizophrenia that are most reliably modelled in mice are the cognitive impairments (67), it will always be impossible to ascribe deficits observed, for example in mice reproducing the $22 q 11$ deletion, to something related to schizophrenia rather than to a fundamental cognitive impairment. Nevertheless, there is interest in the extent to which mouse models of 22q11 deletion syndrome show phenotypes that can be related to schizophrenia (S72,S73). Mice reproducing the 15q13.3 microdeletion (S74) and the $16 \mathrm{p} 11.2$ duplication (S75) have been generated, and should be investigated for disease-relevant phenotypes, using translational models and assays (67). For studies at the cellular level, induced pluripotent stem cells (iPSCs) of course have substantial translational promise as well.

\section{Conclusions}

It will be evident from the above that, despite the general lack of overwhelming evidence implicating individual genes in disease risk, a picture is emerging that is consistent with dysfunctional glutamatergic signalling in schizophrenia. In many ways this should not come as a surprise, given our current (albeit imperfect) understanding of the neurobiology of the disease. In the future, hypothesis-driven epistatic studies should be highly informative for understanding how the individual genetic elements combine to elevate disease risk. Importantly, the genetic evidence does suggest possible avenues for therapeutic investigation. Hypothesis-driven neurobiology studies are urgently required to explore these possibilities, since only once the nature and direction of neurochemical dysfunction is understood can appropriate treatment strategies be refined. In recent years, progress in the genetic aspects of schizophrenia has exceeded progress in the neurobiological aspects, and understanding in both areas is required for the development of improved treatments. 


\section{Figure legends}

\section{Figure 1}

Schematic diagram showing genes with roles at glutamatergic synapses that are implicated in risk for schizophrenia. Main panel illustrates interrelationships between the products of these genes, while corresponding dark shading in small panels indicates evidence from candidate gene studies, GWAS, CNV studies or exome sequencing (see text for further details).

\section{Figure 2}

Schematic diagram showing genes with roles in post-synaptic JNK signalling that are implicated in risk for schizophrenia. Main panel illustrates interrelationships between the products of these genes, while corresponding dark shading in small panels indicates evidence from candidate gene studies, GWAS, CNV studies or exome sequencing (see text for further details).

\section{Figure 3}

Schematic diagram summarising protein interactions of RAC1 and TAK1 with relevance for schizophrenia. Symbols indicate whether current knowledge suggests the influence of common or rare variants (although in the majority of cases functional mutations have not yet been identified, and so this is not totally unequivocal). See text for details.

\section{Table 1}

Summary of CNVs robustly associated with schizophrenia. In each case, genes within the CNV that are functionally related either to glutamate synapses or post-synaptic kinase signalling are highlighted, along with other genes of interest. We include genes of the phosphatidylinositol glycan biosynthesis (PIG) family in the latter category. These genes contribute to the synthesis of glycosylphosphatidylinositol, which anchors a variety of proteins to the cell surface. PIG genes have recently been linked to other CNS disorders $(\mathrm{S} 76, \mathrm{S77})$. There are 21 PIG genes, so although there is no direct evidence linking glycosylphosphatidylinositol anchors to schizophrenia, the presence of 4 of these genes within the CNVs associated with schizophrenia is remarkable. WBS: Williams-Beuren syndrome; Ang/PW: Angelman syndrome, Prader-Willi syndrome. 
Table 1

\begin{tabular}{|c|c|c|c|c|c|}
\hline & Citation & $\begin{array}{l}\text { No. of } \\
\text { genes }\end{array}$ & $\begin{array}{c}\text { Candidate gene } \\
\text { Glutamate } \\
\text { synapse }\end{array}$ & $\begin{array}{l}\text { Candidate gene } \\
\text { Post-synaptic } \\
\text { signalling }\end{array}$ & $\begin{array}{c}\text { Other notable } \\
\text { gene }\end{array}$ \\
\hline 1p36.33 duplication & (37) & 5 & & PRKCZ & GABRD \\
\hline 1q21.1 deletion & $(29,31,59)$ & $\sim 14$ & & PRKAB2 & PDZK1 \\
\hline $2 \mathrm{p} 16.3$ deletion & (S78) & 1 & NRXN1 & & \\
\hline 2q13 duplication & $(36,68)$ & 10 & & MERTK & \\
\hline $3 q 29$ deletion & $(5,17,29)$ & 19 & DLG1, PAK2 & & PIGX, PIGZ \\
\hline $\begin{array}{l}\text { 7q11.23 (WBS) } \\
\text { duplication }\end{array}$ & $(5,31,69)$ & 25 & STX1A, LIMK1 & FZD9 & \\
\hline 7q36.3 duplication & (30) & 1 & & VIPR2 & \\
\hline 9p11.2 deletion & (37) & 2 & SLC1A1 & & \\
\hline 10q23 duplication & (5) & & GRID1 & NRG3 & \\
\hline 15q11.2 deletion & (29)(S79) & $\sim 4$ & CYFIP1 & & \\
\hline $\begin{array}{l}\text { 15q11-q13 } \\
\text { duplication (Ang/PW) }\end{array}$ & $(5,31)(S 80)$ & 15 & & & $\begin{array}{l}\text { GABRA5, } \\
\text { GABRB3, GABRG3 }\end{array}$ \\
\hline 15q13.3 deletion & (29) & 12 & CHRNA7 & ARHGAP11B & \\
\hline 16p13.11 duplication & (5) & 8 & & & NDE1 \\
\hline 16p12.1 deletion & (37) & 7 & & & \\
\hline $\begin{array}{l}\text { 16p11.2 distal } \\
\text { deletion }\end{array}$ & $(37)(S 81)$ & 10 & & $\angle A T$ & \\
\hline 16p11.2 duplication & (55) & 29 & & TAOK2 & $\begin{array}{l}\text { KCTD13, } \\
\text { MAPK3 (ERK1) }\end{array}$ \\
\hline 17p12 deletion & (S79) & 10 & & & PIGL \\
\hline 17q12 del/dupl & (31)(S57) & 18 & & DUSP14 & PIGW, LHX1 \\
\hline 17q21.31 dupl & (5) & 18 & & & CRHR1 \\
\hline 22q11 deletion & $(5)(S 82, S 83)$ & $35-60$ & & $C R K L, T X N R D 2$ & $\begin{array}{l}\text { COMT, PIK4CA, } \\
\text { PRODH, DGCR8 }\end{array}$ \\
\hline
\end{tabular}




\section{References}

\section{See supplementary information for additional references}

1. Tandon R, Keshavan MS, Nasrallah HA. Schizophrenia, "Just the Facts": What we know in 2008: Part 1: Overview. Schizophr Res 2008: 100: 4-19.

2. Millar JK, Wilson-Annan JC, Anderson S et al. Disruption of two novel genes by a translocation cosegregating with schizophrenia. Human Molecular Genetics 2000: 9: 1415-1423.

3. Doherty JL, O'Donovan MC, Owen MJ. Recent genomic advances in schizophrenia. Clin Genet 2012: 81: 103-109.

4. Fromer M, Pocklington AJ, Kavanagh DH et al. De novo mutations in schizophrenia implicate synaptic networks. Nature 2014: 506: 179-184.

5. Kirov G, Rees E, Walters JTR et al. The Penetrance of Copy Number Variations for Schizophrenia and Developmental Delay. Biol Psychiatry 2014: 75: 378-385.

6. Owen MJ. Implications of Genetic Findings for Understanding Schizophrenia. Schizophr Bull 2012: 38: 904-907.

7. Morris BJ, Cochran SM, Pratt JA. PCP: from pharmacology to modelling schizophrenia. Current Opinion in Pharmacology 2005: 5: 1-6.

8. Pratt JA, Winchester CW, Egerton A et al. Modelling prefrontal cortex deficits in schizophrenia: implications for treatment. BrJPharmacol 2008: 153: S465-470.

9. Glessner JT, Reilly MP, Kim CE et al. Strong synaptic transmission impact by copy number variations in schizophrenia. Proc. Natl. Acad.Sci. 2010: 107: 10584-10589.

10. Kirov $G$, Pocklington AJ, Holmans $P$ et al. De novo CNV analysis implicates specific abnormalities of postsynaptic signalling complexes in the pathogenesis of schizophrenia. Mol Psychiatry 2012: 17: 142-153.

11. Purcell SM, Moran JL, Fromer $\mathrm{M}$ et al. A polygenic burden of rare disruptive mutations in schizophrenia. Nature 2014: 506: 185-190.

12. Schizophrenia Working Group of the Psychiatric Genomics C. Biological insights from 108 schizophrenia-associated genetic loci. Nature 2014: 511: 421-427.

13. Winchester CL, Pratt JA, Morris BJ. Risk genes for schizophrenia: Translational opportunities for drug discovery. Pharmacology \& Therapeutics 2014: 143: 34-50.

14. Coffey ET. Nuclear and cytosolic JNK signalling in neurons. Nat Rev Neurosci 2014: 15: 285-299.

15. Pickard BS, Malloy MP, Christoforou A et al. Cytogenetic and genetic evidence supports a role for the kainate-type glutamate receptor gene, GRIK4, in schizophrenia and bipolar disorder. Mol Psychiatry 2006: 11: 847-857.

16. Carroll LS, Williams HJ, Walters J et al. Mutation screening of the $3 q 29$ microdeletion syndrome candidate genes DLG1 and PAK2 in schizophrenia. American journal of medical genetics Part B, Neuropsychiatric genetics 2011: 156B: 844-849.

17. Mulle JG, Dodd AF, McGrath JA et al. Microdeletions of 3q29 Confer High Risk for Schizophrenia. The American Journal of Human Genetics 2010: 87: 229-236.

18. Walsh T, McClellan JM, McCarthy SE et al. Rare Structural Variants Disrupt Multiple Genes in Neurodevelopmental Pathways in Schizophrenia. Science 2008: 320: 539-543.

19. Uhlhaas PJ, Singer W. Abnormal neural oscillations and synchrony in schizophrenia. Nat Rev Neurosci 2010: 11: 100-113.

20. Vinkers $\mathrm{CH}$, Mirza NR, Olivier $\mathrm{B}$ et al. The inhibitory GABA system as a therapeutic target for cognitive symptoms in schizophrenia: investigational agents in the pipeline. Expert Opin Investig Drugs 2010: 19: 1217-1233.

21. Buizer-Voskamp JE, Muntjewerff JW, Strengman E et al. Genome-wide analysis shows increased frequency of copy number variation deletions in Dutch schizophrenia patients. Biol Psychiatry 2011: 70: 655-662.

22. Freedman R. alpha7-nicotinic acetylcholine receptor agonists for cognitive enhancement in schizophrenia. Annual review of medicine 2014: 65: 245-261.

23. Green EK, Grozeva D, Jones I et al. The bipolar disorder risk allele at CACNA1C also confers risk of recurrent major depression and of schizophrenia. Mol Psychiatry 2010: 15: 1016-1022. 
24. Ripke S, Consortium SPG-WASG. Genome-wide association study identifies five new schizophrenia loci. Nat Genet 2011: 43: 969-976.

25. Ripke $\mathrm{S}, \mathrm{O}$ 'Dushlaine $\mathrm{C}$, Chambert $\mathrm{K}$ et al. Genome-wide association analysis identifies 13 new risk loci for schizophrenia. Nat Genet 2013: 45: 1150-1159.

26. Patil ST, Zhang L, Martenyi $F$ et al. Activation of $m G l u 2 / 3$ receptors as a new approach to treat schizophrenia: a randomized Phase 2 clinical trial. Nature Medicine 2007: 13: 1102-1107.

27. Adams D, Kinon B, Baygani S et al. A long-term, phase 2, multicenter, randomized, open-label, comparative safety study of pomaglumetad methionil (LY2140023 monohydrate) versus atypical antipsychotic standard of care in patients with schizophrenia. BMC Psychiatry 2013: 13: 143.

28. Timms AE, Dorschner MO, Wechsler J et al. Support for the N-methyl-D-aspartate receptor hypofunction hypothesis of schizophrenia from exome sequencing in multiplex families. JAMA Psychiatry 2013: 70: 582-590.

29. Levinson DF, Duan J, Oh S et al. Copy Number Variants in Schizophrenia: Confirmation of Five Previous Findings and New Evidence for $3 q 29$ Microdeletions and VIPR2 Duplications. Am J Psychiatry 168, 302-316, 2011.

30. Vacic V, McCarthy S, Malhotra D et al. Duplications of the neuropeptide receptor gene VIPR2 confer significant risk for schizophrenia. Nature 2011: 471: 499-503.

31. Rees E, Walters JTR, Georgieva L et al. Analysis of copy number variations at 15 schizophreniaassociated loci. The British Journal of Psychiatry 2014: 204: 108-114.

32. Hu X, Zhang B, Liu W et al. A survey of rare coding variants in candidate genes in schizophrenia by deep sequencing. Mol Psychiatry 2013: 19: 858-859.

33. Girard SL, Gauthier J, Noreau A et al. Increased exonic de novo mutation rate in individuals with schizophrenia. Nat Genet 2011: 43: 860-863.

34. Need Anna C, McEvoy Joseph P, Gennarelli M et al. Exome Sequencing Followed by Large-Scale Genotyping Suggests a Limited Role for Moderately Rare Risk Factors of Strong Effect in Schizophrenia. The American Journal of Human Genetics 2012: 91: 303-312.

35. Shi J, Levinson DF, Duan J et al. Common variants on chromosome 6 p22.1 are associated with schizophrenia. Nature 2009: 460: 753-757.

36. Costain $\mathrm{G}$, Lionel AC, Fu F et al. Adult neuropsychiatric expression and familial segregation of $2 \mathrm{q} 13$ duplications. American Journal of Medical Genetics Part B: Neuropsychiatric Genetics 2014: 165: 337-344.

37. Rees E, Walters JT, Chambert KD et al. CNV analysis in a large schizophrenia sample implicates deletions at 16p12.1 and SLC1A1 and duplications at 1p36.33 and CGNL1. Hum Mol Genet 2014: 23: 16691676.

38. Myles-Worsley M, Tiobech J, Browning SR et al. Deletion at the SLC1A1 glutamate transporter gene co-segregates with schizophrenia and bipolar schizoaffective disorder in a 5-generation family. American Journal of Medical Genetics Part B: Neuropsychiatric Genetics 2013: 162: 87-95.

39. Millar JK, Pickard BS, Mackie S et al. DISC1 and PDE4B Are Interacting Genetic Factors in Schizophrenia That Regulate cAMP Signaling. Science 2005: 310: 1187-1191.

40. O'Kane EM, Stone TW, Morris BJ. Distribution of Rho family GTPases in the adult rat hippocampus and cerebellum. Mol Brain Res 2003: 114: 1-8.

41. O'Kane EM, Stone TW, Morris BJ. Activation of Rho GTPases by synaptic transmission in the hippocampus. J Neurochem 2003: 87: 1309-1312.

42. Camargo LM, Collura V, Rain J-C et al. Disrupted in Schizophrenia 1 Interactome: evidence for the close connectivity of risk genes and a potential synaptic basis for schizophrenia. Mol Psychiatry 2006: 12: 74-86.

43. Zhao $Q, L i T$, Zhao $X$ et al. Rare CNVs and tag SNPs at $15 q 11.2$ are associated with schizophrenia in the Han Chinese population. Schizophr Bull 2013: 39: 712-719.

44. Xu B, lonita-Laza I, Roos JL et al. De novo gene mutations highlight patterns of genetic and neural complexity in schizophrenia. Nat Genet 2012: 44: 1365-1369.

45. Xu B, Woodroffe A, Rodriguez-Murillo $L$ et al. Elucidating the genetic architecture of familial schizophrenia using rare copy number variant and linkage scans. Proc Natl Acad Sci U S A 2009: 106: 16746-16751. 
46. Need AC, Ge D, Weale ME et al. A Genome-Wide Investigation of SNPs and CNVs in Schizophrenia. PLoS Genet 2009: 5: e1000373.

47. Wong EH, So HC, Li M et al. Common Variants on Xq28 Conferring Risk of Schizophrenia in Han Chinese. Schizophr Bull 2013: 40: 777-786.

48. Ohi K, Hashimoto R, Nakazawa T et al. The p250GAP Gene Is Associated with Risk for Schizophrenia and Schizotypal Personality Traits. PLoS ONE 2012: 7: e35696.

49. Hayashi-Takagi A, Araki Y, Nakamura M et al. PAKs inhibitors ameliorate schizophrenia-associated dendritic spine deterioration in vitro and in vivo during late adolescence. Proc. Natl. Acad.Sci. 2014: 111: 6461-6466.

50. Wang $Q$, Charych EI, Pulito VL et al. The psychiatric disease risk factors DISC1 and TNIK interact to regulate synapse composition and function. Mol Psychiatry 2011: 16: 1006-1023.

51. Hussain NK, Hsin H, Huganir RL et al. MINK and TNIK Differentially Act on Rap2-Mediated Signal Transduction to Regulate Neuronal Structure and AMPA Receptor Function. J Neurosci 2010: 30: 1478614794.

52. Coba MP, Komiyama NH, Nithianantharajah J et al. TNiK Is Required for Postsynaptic and Nuclear Signaling Pathways and Cognitive Function. Journal of Neuroscience 2012: 32: 13987-13999.

53. Potkin SG, Turner JA, Fallon JA et al. Gene discovery through imaging genetics: identification of two novel genes associated with schizophrenia. Mol Psychiatry 2008: 14: 416-428.

54. Zheng $\mathrm{X}$, Bei J-X, Xu H et al. The association between rare large duplication of $16 \mathrm{p} 11.2$ and schizophrenia in the Singaporean Chinese population. Schizophr Res 2013: 146: 368-369.

55. McCarthy SE, Makarov V, Kirov G et al. Microduplications of 16p11.2 are associated with schizophrenia. Nat Genet 2009: 41: 1223-1227.

56. Addington AM, Rapoport JL. The genetics of childhood-onset schizophrenia: when madness strikes the prepubescent. Current psychiatry reports 2009: 11: 156-161.

57. Steinberg S, de Jong S, Mattheisen M et al. Common variant at $16 \mathrm{p} 11.2$ conferring risk of psychosis. Mol Psychiatry 2012.

58. Van Den Bossche MJ, Strazisar M, Cammaerts $S$ et al. Identification of rare copy number variants in high burden schizophrenia families. American Journal of Medical Genetics Part B: Neuropsychiatric Genetics 2013: 162: 273-282.

59. Stefansson $\mathrm{H}$, Rujescu $\mathrm{D}$, Cichon $\mathrm{S}$ et al. Large recurrent microdeletions associated with schizophrenia. Nature 2008: 455: 232-236.

60. Li M, Wang $\mathrm{Y}$, Zheng $\mathrm{X}$-b et al. Meta-analysis and brain imaging data support the involvement of VRK2 (rs2312147) in schizophrenia susceptibility. Schizophr Res 2012: 142: 200-205.

61. Steinberg $S$, de Jong $S$, Andreassen OA et al. Common variants at VRK2 and TCF4 conferring risk of schizophrenia. Hum Mol Genet 2011: 20: 4076-4081.

62. Winchester $\mathrm{CL}$, Ohzeki $\mathrm{H}$, Vouyiouklis $\mathrm{DA}$ et al. Converging evidence that sequence variations in the novel candidate gene MAP2K7 (MKK7) are functionally associated with schizophrenia. Human Molecular Genetics 2012: 21: 4910-4921.

63. Bassett AS, Costain G, Alan Fung WL et al. Clinically detectable copy number variations in a Canadian catchment population of schizophrenia. J Psychiatr Res 2010: 44: 1005-1009.

64. Yamasaki T, Kawasaki H, Arakawa S et al. Stress-Activated Protein Kinase MKK7 Regulates Axon Elongation in the Developing Cerebral Cortex. The Journal of Neuroscience 2011: 31: 16872-16883.

65. Fuller G, Veitch K, Lai Kwan H et al. Activation of p44/p42 MAP kinase in striatal neurons via kainate receptors and PI3 kinase. Molecular Brain Research 2001: 89: 126-132.

66. Clark CJ, McDade DM, O'Shaughnessy CT et al. Contrasting roles of neuronal Msk1 and Rsk2 in Bad phosphorylation and feedback regulation of Erk signalling. J Neurochem 2007: 102: 1024-1034.

67. Pratt JA, Winchester C, Dawson N et al. Advancing schizophrenia drug discovery: optimizing rodent models to bridge the translational gap. Nat Rev Drug Discovery 2012: 11: 560-579.

68. Costain G, Lionel AC, Fu F et al. Adult neuropsychiatric expression and familial segregation of $2 q 13$ duplications. American Journal of Medical Genetics Part B: Neuropsychiatric Genetics 2014: n/a-n/a.

69. Mulle JG, Pulver AE, McGrath JA et al. Reciprocal duplication of the Williams-Beuren syndrome deletion on chromosome 7q11.23 is associated with schizophrenia. Biol Psychiatry 2014: 75: 371-377. 